\title{
Pénurie de main-d'œuvre et dispositifs localisés d'insertion dans les pêches maritimes en Bretagne
}

To guide job seekers to employment in fishing industry in Brittany: local insertion policies

\section{Annie Gouzien}

\section{(2) OpenEdition}

\section{Journals}

Édition électronique

URL : http://journals.openedition.org/travailemploi/3469

DOI : $10.4000 /$ travailemploi.3469

ISSN : 1775-416X

Éditeur

DARES - Ministère du Travail

Édition imprimée

Date de publication : 30 septembre 2009

Pagination : 23-35

ISSN : 0224-4365

Référence électronique

Annie Gouzien, «Pénurie de main-d'œuvre et dispositifs localisés d'insertion dans les pêches maritimes en Bretagne », Travail et Emploi [En ligne], 119 | juillet-septembre 2009, mis en ligne le 30 septembre 2011, consulté le 01 mai 2019. URL : http://journals.openedition.org/travailemploi/3469 ; DOl : 10.4000/travailemploi.3469 


\title{
Pénurie de main-d'œuvre et dispositifs localisés d'insertion dans les pêches maritimes en Bretagne
}

\author{
Annie Gouzien (*)
}

\begin{abstract}
Le présent article analyse les conditions, pratiques et institutions de l'accompagnement vers l'emploi dans la socio-économie des pêches maritimes en Bretagne. Il cherche à rendre compte du processus de passage, des formes traditionnelles d'accès à l'emploi portées par le milieu, à des formes plus contemporaines fondées sur les recherches de connexions entre les pratiques et institutions propres au secteur et celles du Service public de l'emploi. Il analyse dans un premier temps la diversité des formes historiques d'accompagnement par le milieu selon différents quartiers maritimes; dans un second temps, il rend compte des conditions institutionnelles qui ont permis, dans différents domaines, l'ouverture du secteur sur la société globale; enfin il termine par l'observation fine des modes de construction interactionnistes d'un dispositif localisé d'insertion de demandeurs d'emploi vers la socio-économie des pêches.
\end{abstract}

La notion d'accompagnement vers l'emploi désigne en général les pratiques professionnelles de suivi individualisé de demandeurs d'emploi dans le cadre de dispositifs publics ou de dispositifs privés faisant par ailleurs l'objet d'un contrôle public. L'analyse de ce champ d'action s'oriente donc assez naturellement vers les institutions spécialisées chargées de cette mission ( $c f$. BALZANI et al., 2008) qui, dans un contexte de développement du chômage structurel, se sont considérablement développées et complexifiées. Il est sans doute moins courant d'utiliser la notion pour parler des caractéristiques «porteuses» d'un milieu socio-économique dont la fonction d'insertion professionnelle de sa population active a été longtemps faiblement institutionnalisée (Gouzien, 2008). Dans le secteur des pêches maritimes français, l'accès à l'emploi relève historiquement d'un accompagnement «par le milieu». La fonction de placement ou d'insertion professionnelle est largement encastrée dans l'ensemble des échanges qui constituent la socio-économie sectorielle. L'institutionnalisation du placement, quand elle existe, reste interne au secteur d'activité et entretient peu de liens avec le Service public de l'emploi. Cependant, depuis la dernière grande crise de la pêche datant des années 1990, ce modèle d'autoreproduction professionnelle et de relative fermeture sectorielle donne des signes persistants de faiblesse dont un des symptômes majeurs est la pénurie de main-d'œuvre qui contribue à asphyxier l'activité économique des flottilles de pêche. La profession se mobilise alors pour résoudre le problème d'affai-

(*) CRESS Lessor, Rennes 2; annie.gouzien@uhb.fr blissement de ses viviers propres et attirer vers ses entreprises de pêche une main-d'oeuvre extérieure au milieu.

C'est dans ce contexte que la problématique de l'accompagnement vers l'emploi, au premier sens de la définition proposée en introduction, se trouve appropriée par des acteurs du milieu des pêches. Ce sont les entreprises de pêches, ou plus particulièrement les institutions sectorielles qui les représentent, qui se retrouvent initiatrices des dispositifs d'accompagnement. On pourrait avancer que c'est une logique de l'offre d'emploi, celle des entreprises, qui porte cette dynamique, et non une logique de la demande d'emploi, dominante dans les institutions publiques de gestion des demandeurs d'emploi. Cette posture originale interroge les conditions nécessaires pour que les «connexions» s'établissent entre les modalités traditionnelles, historiques, de l'accompagnement par le milieu, et celles, plus contemporaines, du champ des politiques actives de l'emploi. Pour rendre compte de ce processus (voir encadré 1), nous procéderons en trois temps. En premier lieu, nous rendrons compte des pratiques et institutions de l'accompagnement vers l'emploi dans le milieu des pêches maritimes en Bretagne en adoptant une posture socio-économique restituant comment la diversité des ancrages de l'activité économique des pêches dans les dimensions sociales et politiques locales contribuent à construire cet accompagnement. Ensuite nous restituerons les innovations institutionnelles diverses et progressives produites par le milieu des pêches, depuis la crise des années 1990, pour favoriser une meilleure coordination avec les institutions globales. Enfin, nous montrerons comment ces 


\section{Encadré 1}

\section{Éléments de cadrage théorique}

Nous nous appuierons sur trois référentiels théoriques pour rendre compte de l'accompagnement vers l'emploi dans le milieu des pêches maritimes et de ses évolutions. La sociologie économique considère "l'économie comme une totalité sociale" et non comme un "champ d'étude plus restreint relevant d'une conception endogène des phénomènes économiques dont les processus sont interprétés comme s'ils se réalisaient selon la seule logique pure de l'action rationnelle" (LeVESQUE, BouRgues, Forgues, 2001). Dans cet esprit nous cherchons à rendre compte de la façon dont les pratiques d'accès à l'emploi dans le milieu des pêches ainsi que ses institutions s'insèrent dans un système social complexe qu'exprime la notion d'accompagnement par le milieu. Considérant l'économie comme une construction sociale, nous accordons une attention particulière à la façon dont les pratiques économiques des acteurs s'inscrivent dans des modèles sociaux qui se cristallisent dans des institutions contribuant en retour à orienter leur action (BERGER, LUCKMAN, 1989). Ceci nous amène à accorder une attention particulière aux transformations institutionnelles qui accompagnent les tentatives d'ouverture du secteur des pêches à la société globale pour résoudre les problèmes de pénurie de main-d'oeuvre. Enfin, pour rendre compte de l'appropriation par les acteurs professionnels du milieu des pêches des problématiques d'accompagnement, au sens des politiques actives de l'emploi, nous mobiliserons la conception interactionniste d'élaboration des institutions qui accorde une attention particulière aux modes de coordination qui s'établissent entre les acteurs en interaction. Nous utiliserons à cet effet la notion de «relation localisée d'insertion» proposée par Simon WUHL (1996) pour rendre compte de l'expérience d'accompagnement vers l'emploi dans le secteur des pêches menée notamment par l'Association Cap Avenir.

recherches de coordination entre dynamiques endogènes du milieu et institutions publiques nationales supposent une construction de type interactionniste. Celle-ci débouche sur des systèmes d'insertion localisés susceptibles d'étoffer le maillage socioinstitutionnel local d'accès à l'emploi et assure de ce fait un meilleur "portage» des parcours des demandeurs d'emploi extérieurs au milieu.

\section{Socio-économie des pêches maritimes, mode de gestion de l'emploi et accompagnement par le milieu}

Les mobilités d'emploi et l'insertion professionnelle des marins pêcheurs dans la socio-économie des pêches maritimes bretonnes sont indissociables des spécificités de quartiers maritimes. Les pêches maritimes bretonnes sont organisées en systèmes productifs localisés (Courlet, PeCQuert, 1992) différenciés entre eux sur la base d'identités socioéconomiques particulières. Les mobilités d'emploi et les modes d'accès à l'emploi sont supportés par un faisceau de relations complexes obéissant à des rapports de type systémique entre caractéristiques des flottilles, des systèmes de formation, des marchés localisés du travail et des systèmes de gouvernance professionnelle propres à chaque quartier maritime (Gouzien, 2005, 2007). Ainsi la «gestion locale de l'emploi» dépend des capacités de recrutement des lycées maritimes qui forment les futurs marins pêcheurs, mais aussi des régulations des mobilités de main-d'oeuvre propres aux différents quartiers. Ces mobilités obéissent assez largement à des relations d'interconnaissance entre marins fondées sur la réputation professionnelle à l'image des districts industriels (BecCatini, Trigilia, 1988). Ces modes de recrutement «porto-portuaires» spontanés sont toutefois complétés, variablement selon les quartiers, par des Bourses maritimes qui assurent le rapprochement des offres et des demandes d'embarquement quand les pratiques porto-portuaires ne sont plus suffisantes; ce qui devient effectif à partir des années 1980.

\section{La socio-économie des pêches maritimes: des systèmes productifs localisés à fortes identités territoriales}

La socio-économie des pêches maritimes en Bretagne est d'abord identifiable spatialement sous la forme d'une grande diversité de ports et de flottilles. La différenciation géographique de l'activité des pêches tient son origine du découpage administratif du bord de côte en quartiers maritimes. Par la suite, les communautés de pêches se sont approprié ces territoires portuaires en les spécifiant à partir des ressources qui leur étaient disponibles. Selon les ressources naturelles des quartiers, les capacités entrepreneuriales des marins, la richesse ou la solidité du tissu social porteur, les capitaux disponibles, ces communautés locales ont coloré différemment ces ports de pêche. Les identités des quartiers ont varié durant l'histoire. Au gré des contraintes de contexte, des trajectoires portuaires se sont modifiées. Des ports ont périclité, d'autres se sont développés. Les modèles sociaux d'activité ont également évolué entre le modèle artisan, toujours dominant en Bretagne, et le modèle semi-industriel ou industriel qui s'est plutôt affaibli depuis les années 1990.

Toutefois, dans l'ensemble, les quartiers maritimes ont cherché à préserver leur identité économique particulière car celle-ci est à la fois cause et effet de l'ancrage territorial de l'activité économique et de sa performance. Trois quartiers mari- 
times typiques des ports bretons illustrent particulièrement cette diversité. Le Quartier de Paimpol en Bretagne nord est un quartier de petite pêche spécialisé sur la coquille saint-jacques; la moitié des marins pêcheurs occupe de ce fait un emploi de patron pêcheur. Il dispose d'un lycée maritime qui couvre ses besoins de formation ainsi que ceux des quartiers avoisinants au profil plus hauturier comme celui de St.-Brieuc. Le Quartier de Concarneau en Bretagne sud est historiquement un quartier à dominante de pêche industrielle et semi-industrielle. Ses flottilles capturent une grande variété de poissons depuis les poissons de grands fonds pêchés par les chalutiers semi-industriels, en passant par les poissons bleus et les crustacés, capturés par les thoniers océaniques de grande pêche pour le thon et par les côtiers artisans pour la langoustine. Son marché du travail est fortement différencié et segmenté selon les flottilles. La pénurie de main-d'oeuvre touche plus particulièrement les matelots des armements industriels. Le Quartier de Concarneau ne dispose plus depuis les années 1980 de lycée maritime, en revanche il bénéficie d'un Centre de formation européen pour la formation professionnelle continue des marins pêcheurs. Enfin le Quartier du Guilvinec est toujours considéré comme le premier quartier de pêche artisanale au plan national. Ses quatre ports offrent une grande diversité de poissons et de crustacés. Il dispose d'un lycée maritime qui ne pourvoit plus suffisamment à ses besoins en nouveaux entrants. Il se trouve régulièrement confronté à des fortes tensions en matière de recrutement qui obligent parfois ses navires à rester à quai.

\section{Autoreproduction professionnelle et accompagnement par le milieu}

À la relative intégration fonctionnelle territorialisée des institutions correspondent des modèles sociaux, des cultures locales, des façons de faire, d'agir et de penser, produisant des formes endogènes de production, de reproduction et de circulation sociales dans le milieu. La notion d'accompagnement par le milieu renvoie à l'ensemble de ces facettes. Elle désigne l'existence d'une densité d'interactions qui constitue un tissu socio-économique dont les modalités d'action demeurent encore fortement endogènes et différenciées selon les quartiers maritimes. Chacun dispose de ses modèles et normes sociales qui guident et orientent la mobilité des marins pêcheurs. Jusqu'à une période encore récente, l'orientation des populations maritimes vers cette activité se produisait sur la base d'une proximité géographique (déversement des viviers agricoles répondant aux besoins en main-d'œuvre du secteur des pêches maritimes en période faste de développement), d'une proximité sociale (orientations familiales ou par le voisinage des candidats à l'embarquement) et d'une proximité institutionnelle (orientation, familiale des jeunes, vers les écoles de pêches, et, professionnelle, des marins adultes, vers la formation continue, en fonction des besoins du système d'activité). C'est l'ensemble de la communauté sociale des pêcheurs, dans des lieux faiblement dédiés à la fonction placement, comme les cafés, les espaces domestiques, de voisinage, les commerces, les pontons, qui se charge de donner l'information et de favoriser la circulation de la population active entre les différentes institutions et lieux de la flottille.

«Ce qui fonctionne le mieux, c'est la filière ellemême, en interne, avec les avantages et inconvénients que ça comporte: c'est un petit peu en circuit fermé et en même temps c'est très efficace. C'est vrai que par le bouche à oreille, il y a une certaine sélection qui se fait(1). »

Un certain nombre de modèles socioculturels partagés constituent les opérateurs de cette circulation: l'importance des compétences de métiers pour s'orienter dans la flottille; les relations orales, de gré à gré, pour s'engager dans l'emploi; les modèles d'activité et salariaux que constituent respectivement l'installation artisanale et le salaire à la part (2). Ces modèles socioculturels contribuent également à la différenciation des quartiers maritimes. Dans les quartiers de petite pêche comme celui de Paimpol le modèle d'activité et d'emploi est historiquement celui de l'installation artisanale fondée sur la transmission patrimoniale familiale; dans le Quartier du Guilvinec le modèle de l'installation artisanale hauturière a été fortement soutenu par le dynamisme des coopératives locales d'armement dans les années 1970-1980 (GouzIEN, 2006) tandis que le Quartier de Concarneau a plutôt porté un modèle d'emploi, fondé sur la carrière salariale dans des armements privés, moins présent dans les quartiers précédents (CÉREQ, 2003). Ces diversités locales des modèles sociaux d'activité et d'emploi s'expriment dans le système de gouvernance professionnelle que constituent les comités professionnels (3) propres à chaque quartier. Leur composition exprime en général la configuration sociologique particulière des métiers de la pêche propres à chaque quartier (Gouzien, 2004).

(1) Permanent du comité régional des pêches maritimes et des élevages marins en Bretagne.

(2) Le modèle artisanal préserve une continuité entre la propriété de l'entreprise et l'activité productive puisque le patron artisan est un armateur embarqué. Cette hybridation capital travail est renforcée par le salaire à la part qui répartit les risques positifs et négatifs de l'entreprise de pêche sur l'ensemble des membres de l'équipage: armateur et salariés.

(3) La socio-économie des pêches dispose de ses instances de gouvernance territorialisées que sont les comités locaux des pêches maritimes et des élevages marins. Ils rassemblent des représentants des armateurs et des marins élus sur listes syndicales ainsi que des représentants de coopératives locales désignés par la Fédération de la coopération maritime ou d'autres acteurs professionnels de la filière comme les mareyeurs. 


\section{Une institution sectorielle du placement: les bourses maritimes de l'emploi}

Dans certains quartiers maritimes, la fonction placement tend toutefois à se spécialiser dans le cadre des comités locaux des pêches maritimes et des élevages marins. Des patrons pêcheurs et des membres d'équipage viennent déposer leurs demandes et offres d'embarquement auprès des secrétaires de ces organisations qui assurent alors le rapprochement. D'autres pratiques existent comme le dépôt de demandes d'embarquement par des marins pêcheurs aux sièges sociaux des armements en société et l'affichage des offres et demandes d'embarquement dans les locaux de l'administration des Affaires maritimes. Ces formes plus spécialisées sont utilisées quand les méthodes les plus courantes, jugées aussi comme étant les plus fiables, comme «le bouche à oreille», ne sont plus suffisantes. À partir des années 1980, l'autoreproduction par le milieu donne des signes d'essoufflement. Les lycées professionnels maritimes ne recrutent plus aussi facilement et l'économie des pêches attire moins les populations actives environnantes. Le déficit de main-d'oeuvre a été mis au compte de phénomènes variés de nature démographique, sociologique et institutionnelle; la capacité du milieu à produire ses propres ressources humaines n'est plus suffisante, tandis que les conditions institutionnelles d'entrée dans l'univers des pêches apparaissent toujours très contraignantes. La question centrale devient alors celle des modes de connexion entre un univers socioproductif fortement spécifié culturellement et institutionnellement et les ressources de la société globale. Les acteurs de l'économie des pêches interpellent plus particulièrement les institutions nationales sur la question de l'emploi et de la formation. L'appropriation de la problématique de l'accompagnement par les acteurs du milieu s'inscrit dans ce contexte. Elle donne lieu, notamment, à des expériences originales de co-construction de dispositifs d'accompagnement individualisé comme celui de «Cap Avenir» dans les Côtes-d'Armor. Elle est précédée, par ailleurs, par un ensemble d'innovations institutionnelles d'origines locales variées, initiées par d'autres quartiers maritimes, aboutissant à renforcer les liens entre le milieu des pêches maritimes et la société globale.

\section{Ouverture du milieu des pêches maritimes et innovations institutionnelles}

La spécificité institutionnelle et culturelle du secteur des pêches maritimes en France contribue à une fermeture sectorielle de ses systèmes d'activité et d'emploi. Dans les années 1990, suite à la dernière grande crise du secteur, le milieu ne peut plus assurer seul sa reproduction professionnelle. La pénurie de main-d'œuvre à laquelle il se trouve confronté le met au défi d'adapter ses institutions pour s'ouvrir aux ressources de la société globale.

\section{Spécificité institutionnelle et fermeture sectorielle}

Le milieu des pêches maritimes en France dispose d'un fort particularisme institutionnel aux ancrages historiques profonds. Il dispose d'une administration de tutelle, celle des Affaires maritimes, qualifiée par Louis Mordrel (1972) d'Administration de synthèse dans la mesure où elle englobe les différentes facettes de l'action administrative du secteur(4). Les administrateurs des Affaires maritimes sont présents, de droit, dans les comités professionnels du secteur, dans les coopératives nombreuses dans l'économie des pêches, dans les institutions sectorielles de la protection sociale (ENIM) (5) et de la formation. En tant que «chefs de quartier», ils administrent le système d'immatriculation des navires et des marins. Ils veillent à la conformité technique des navires et à la sécurité des équipages. Avec le système des décisions d'effectifs (6) et des mesures dérogatoires y afférant, ils déterminent les contours du marché du travail maritime et régulent administrativement les mouvements d'emploi. Ils disposent de toutes les informations sur la carrière du marin nécessaires à la gestion de ses droits sociaux par l'ENIM. Ils assurent également le rôle d'inspecteur du travail et sont les interlocuteurs privilégiés des comités

(4) Elle tient ses origines de l'institution colbertiste de la conscription militaire correspondant à une obligation de service militaire appliquée exclusivement aux populations maritimes pour servir sur les vaisseaux du roi en cas de guerre. Ce statut exorbitant du droit commun appliqué aux «gens de mer» nécessita une activité d'inventaire et de classification des populations maritimes (l'inscription maritime) pour lever les populations au fur et à mesure des besoins de la marine royale. Ce statut exorbitant du droit commun réglait la vie de travail des marins pêcheurs et les modalités de l'activité des pêches. Il prévoit que ce sont des règles de droit public qui s'appliquent à une activité privée. Il se traduit par la présence tutélaire de différents corps d'État dans les institutions professionnelles. Les postes de direction sont occupés par le corps militaire des administrateurs des Affaires maritimes. Cette inscription maritime existe toujours sous la forme du système d'immatriculation des marins.

(5) Établissement national des invalides de la Marine.

(6) Les décisions d'effectifs résident dans la détermination par l'administration des Affaires maritimes du nombre et de la qualité des membres composant l'équipage du navire en fonction de son autorisation de durée d'absence du port de pêche déterminée par son appartenance aux genres de navigation $(\mathrm{GN})$. Ainsi un navire de petite pêche est autorisé à naviguer vingt-quatre heures sans rejoindre un port de pêche et sa décision d'effectif est de 1 à 2 membres d'équipage, dont le niveau de qualification déterminé par la détention de brevets professionnels est précisément spécifiée. Les décisions d'effectif d'une flottille consenties par l'administration des Affaires maritimes contribuent donc à dessiner le contour du système d'emploi et de qualification du quartier maritime. 
professionnels dans toutes les situations de conflit. Le caractère fortement administré du marché du travail à la pêche participe aux tensions qui affectent l'emploi. L'insuffisante adéquation entre la classification des postes de travail et les brevets détenus par les marins peut aboutir à une immobilisation des navires à quai. Ces tensions sont en général gérées souplement grâce au système des dérogations qui permet à l'administration d'autoriser un marin compétent, mais ne possédant pas le brevet, à occuper le poste, en contrepartie d'un engagement de formation professionnelle dans le futur. Le nombre de dérogations attribué par l'administration des Affaires maritimes dans un quartier constitue un indicateur assez fiable des tensions de maind'oeuvre existant sur ce quartier. Ainsi en 2000, pour le Quartier de Concarneau, $83 \%$ des marins ayant bénéficié d'une dérogation sont en pêche au large et en grande pêche (GouZIEN, 2003). Le marché du travail à la pêche peut donc être considéré comme un marché du travail relativement fermé institutionnellement du fait de son caractère professionnel exigeant la détention de brevets pour occuper les postes. Jusqu'en 1989, date de la réforme Loquet du système de formation maritime, ce marché professionnel fonctionne en circuit fermé. L'entrée suppose le passage par la formation initiale. Elle assure la formation de base du marin et débouche sur le certificat d'aptitude professionnelle (CAP). La progression dans la carrière se fait ensuite dans le cadre de la formation professionnelle continue par alternance combinant un temps de formation en école de pêche débouchant sur un diplôme et une période de navigation permettant la validation de ce diplôme en brevet professionnel. Fondé sur le modèle de la promotion sociale, ce système de la formation maritime a été qualifié d'ordre hiérarchique et pyramidal (PARADEISE, 1982) puisque le marin ne peut y faire carrière qu'en ayant obtenu l'ensemble des diplômes de la filière. Cet état de fait rend très difficiles les mobilités avec les autres secteurs professionnels.

\section{Les facteurs économiques, sociologiques et institutionnels de mutation contribuant à l'ouverture du milieu}

Depuis les années 1990, la socio-économie des pêches est confrontée à des mutations socio-économiques et institutionnelles sans précédent. La dernière crise du secteur qui survient en 1993 s'est traduite par une contraction du système d'activité et d'emploi de $40 \%$ en Bretagne, fragilisant considérablement la densité de son tissu social. Cette crise est à la fois économique, sociale et institutionnelle. Sur le plan économique, la rentabilité des entreprises de pêche, notamment des entreprises hautu- rières, est fortement compromise (7) par la triple crise de la ressource halieutique, de l'augmentation des charges d'exploitation et de la tendance à la baisse du prix du poisson en raison d'un recours de plus en plus fort à l'importation. Sur le plan social, les capacités d'autoreproduction professionnelle du milieu se sont affaiblies. Les viviers ruraux se sont considérablement taris et la transmission générationnelle de l'entreprise artisanale de pêche s'est trouvée concurrencée, pour les enfants de patrons pêcheurs, par des modèles alternatifs d'orientation scolaire leur ouvrant l'espace de destinée professionnelle. Sur le plan institutionnel, le modèle des systèmes productifs localisés s'est également affaibli. La réduction du système d'activité s'est traduite par une rupture de l'équilibre économique et spatial entre les différents quartiers, certains se développant au détriment des autres, et par une difficulté à préserver l'ensemble des institutions portuaires. Le système de formation s'est réduit et s'est spécialisé non plus principalement en fonction des spécificités productives de quartier, mais de rationalités budgétaires du système de formation lui-même. La nécessité de réaliser des économies d'échelle a occasionné un partage fonctionnel des formations ne cadrant plus toujours très bien avec les pratiques de proximité des marins pêcheurs. Ainsi le Centre européen de formation professionnelle maritime situé à Concarneau parvient difficilement à attirer les flux des futurs patrons pêcheurs du Guilvinec habitués à suivre leur formation dans le lycée maritime du quartier. De façon plus générale, le système des pêches en Bretagne est confronté à un problème de pénurie de main-d'œuvre qu'il ne peut plus résoudre selon les modalités anciennes. Il lui faut définir les conditions de reproduction de son système d'activité, d'emploi et de formation, en relation plus étroite avec les ressources de la société globale.

\section{Les changements et innovations institutionnels dans le champ de l'emploi, de la formation et de l'insertion}

Dans les quinze dernières années, les acteurs du milieu des pêches maritimes en Bretagne se sont mobilisés dans une grande diversité de domaines pour construire des connexions entre leurs institutions propres et celles de la société globale. Les principaux champs couverts ont été ceux de la formation, du placement, de l'insertion, voire de l'organisation professionnelle. Ces initiatives se sont développées en rapport avec les spécificités locales de quartier prises en compte par les administrations de

(7) Système d'activité fortement subventionné jusqu'aux années 1980, dans le cadre national et européen, il doit dorénavant faire face à une flambée du prix des bateaux et des charges d'exploitation et à une réduction des gains tandis que se réduisent considérablement les aides à l'installation et les subventions publiques. 
tutelle selon le modèle étato-corporatiste propre à la trajectoire publique française (TRIPIER, 1998). Ces innovations épousent les spécificités institutionnelles de quartiers qui soutiennent des conceptions culturelles différentes du changement. Cependant l'accent mis sur la coordination de ces initiatives dans le champ de l'emploi, exprime la recherche de modes d'intégration, horizontaux (territoriaux) et verticaux (sectoriels) de ces initiatives innovantes.

\section{Diversité des champs couverts: formation, placement, insertion, organisation professionnelle}

Les premières initiatives visent une meilleure connexion entre institutions sectorielles et institutions nationales. Elles concernent le système de formation des pêches maritimes. Rappelons que jusqu'en 1989 la formation initiale et professionnelle continue des pêches maritimes se déroule dans les écoles d'apprentissage maritime (EAM). Elles décernent le seul titre de formation professionnelle accessible par la formation initiale: le certificat d'apprentissage maritime (CAM) et l'ensemble des brevets professionnels des filières pont et machine, accessibles uniquement par la voie de la formation continue. Les deux filières de formation reposent sur le modèle de l'alternance entre formation scolaire et formation sur le tas (8). En 1989, la réforme Loquet établit les bases d'une véritable filière de formation initiale à la pêche maritime permettant l'établissement de correspondances entre le système de certification de l'Éducation nationale et la classification sectorielle. Toutefois ces correspondances sont limitées au CAP et au BEP jusqu'en 2006. La mobilisation des acteurs de la Commission formation du comité local des pêches du Quartier maritime du Guilvinec favorise l'élévation de la filière de formation au niveau du bac professionnel. Ils œuvrent encore aujourd'hui activement à l'obtention d'un BTS. Dans d'autres quartiers maritimes, l'accent est plutôt mis sur la réappropriation par les acteurs locaux du modèle de formation par apprentissage. Un CAP par apprentissage est proposé en 2004 par le lycée maritime de Paimpol dans le cadre de la formation initiale, tandis que le Centre européen pour la formation professionnelle continue expérimente en 2002, en partenariat avec la flottille semiindustrielle concarnoise de chalutage de fond, un CAP par apprentissage dans le cadre de la formation professionnelle continue.

Dans le domaine du placement, les initiatives sont plus récentes. Les marins pêcheurs en pêche artisa-

(8) Le jeune en apprentissage ne transforme son certificat d'apprentissage maritime en brevet professionnel de matelot qualifié qu'à l'issue de deux années de noviciat à bord des bateaux. La formation professionnelle continue obéit aux mêmes principes. Les diplômes professionnels obtenus à l'école de pêche dans le cadre de la formation continue ne sont transformés en brevets professionnels qu'à l'issue de périodes bien déterminées de navigation. nale fréquentent peu les institutions de gestion de l'assurance chômage et du placement car ce secteur échappe à cette législation. Celle-ci ne s'exerce que sur les armements en société. En conséquence, les marins pêcheurs artisans en rupture d'embarquement qui souhaitent rester dans le secteur s'inscrivent peu à l'ANPE et font plutôt appel aux institutions et pratiques «porto-portuaires» du milieu. La première tentative d'établissement de liens entre bourse maritime et agence locale pour l'emploi a vu le jour, vers la fin des années 1990, dans le Quartier de Concarneau, pour tenter de résoudre les difficultés de recrutement des armements semiindustriels de chalutage de fond(9). Il est donc assez significatif que ces premières connexions se soient établies dans un système productif localisé caractérisé par le modèle «industriel» des pêches, plus proche du modèle salarial, dans lequel les marins bénéficient de l'assurance chômage. En 2007, l'initiative a été relayée au plan régional par une convention associant le comité régional des pêches maritimes et des élevages marins et l'ANPE régionale. Elle suscite des partenariats localisés sur les questions du placement et de l'insertion professionnelle pour l'ensemble des quartiers maritimes de Bretagne, élargissant ainsi la problématique aux quartiers artisans.

Dans le domaine de l'insertion professionnelle, les premières initiatives ont lieu en 1992 avec l'établissement du certificat d'initiation nautique (CIN) (10). L'enjeu est de desserrer l'effet de clôture professionnelle qu'induit l'obligation d'être détenteur d'un CAP pour accéder aux marchés du travail à la pêche maritime. En 2004, les professionnels des pêches des quartiers cornouaillais intègrent au CIN un module de ramendage permettant une meilleure prise en compte des compétences professionnelles nécessaires aux métiers du chalut dominant en Bretagne sud et le rebaptisent certificat d'initiation pêche (CIP). Enfin, tout récemment en 2007, le CIN devient accessible, pour la pêche, par la voie d'un contrat de qualification, à l'initiative de quartiers maritimes du nord de la Bretagne sous l'appellation du contrat de professionnalisation (LE TREUST, 2007). C'est dans le cadre de la mise en œuvre de ces contrats de professionnalisation, notamment, que se développe l'offre d'accompagnement par le milieu.

Dans le domaine de l'organisation professionnelle, l'ensemble de ces initiatives trouve un point

(9) Cette expérimentation a pris la forme d'un rapprochement et d'une coordination entre bourse maritime et ALE s'engageant à s'informer mutuellement de l'état des offres et des demandes d'embarquement.

(10) Il s'adresse aux demandeurs d'emploi sans titre maritime âgés d'au moins 20 ans. Il s'agit d'une formation courte de huit semaines centrée sur les savoirs élémentaires de la navigation, de la sécurité à bord des bateaux et du travail dans un environnement maritime. 
d'aboutissement dans la réflexion relative à l'établissement d'une fonction spécialisée de coordination de la relation formation-emploi pour le secteur des pêches. Différents acteurs du milieu des pêches se sont mobilisés pour contribuer à assurer cette coordination sectorielle ou pour inscrire la question de son existence dans l'agenda public. On peut citer, de façon non exhaustive, le projet présenté dans le cadre des Assises régionales de la pêche par le CLPMEM du Guilvinec intitulé «Un conseiller emploi-formation en Finistère pour la filière pêche», ainsi que les pratiques en œuvre dans le cadre de l'association Cap Avenir pour la Bretagne nord. D'autres institutions, sectorielle comme l'Observatoire de la relation formation-emploi assurée par le FAF Pêche et Cultures marines ou territoriale comme le comité de bassin d'emploi du pays bigouden, de Douarnenez et du Cap Sizun(11), assurent la production de connaissance statistique et la fonction d'études sur les dynamiques des marchés du travail à la pêche maritime.

\section{Les conditions de connexion entre les institutions sectorielles et celles de la société globale}

Les innovations institutionnelles s'inscrivent dans une imbrication serrée des échelles d'intervention entre le niveau local et national. Les niveaux locaux des quartiers maritimes font preuve de dynamisme et d'originalité. Ils recherchent des adaptations ou des innovations en rapport avec les caractéristiques culturelles et productives de leurs systèmes productifs locaux. Parfois ces innovations renvoient à des expérimentations locales qui feront l'objet d'extension à un niveau géographique supérieur (cas de rapprochement entre ALE et bourses maritimes et de la création du CIP). D'autres fois, des initiatives nationales sont adaptées localement (cas du CIN, des contrats de professionnalisation, du CAP par apprentissage). D'autres fois encore, des quartiers maritimes s'inscrivent dans des cadres législatifs rénovateurs, auxquels ils ont probablement activement contribué, pour enrichir la filière de formation et la qualification des emplois et de la main-d'œuvre (cas de la construction de la filière de formation initiale à la pêche maritime). Ces orientations apparemment contradictoires ne peuvent être comprises indépendamment des modèles institutionnels de relations qui s'établissent entre les acteurs professionnels des pêches et les acteurs publics, dont ceux de l'administration des Affaires maritimes. On peut penser que cette administration de synthèse, contribuant par la particularisation du milieu à sa clôture institutionnelle, participe toutefois, du fait de la présence de ses acteurs aux différents échelons des instances de gouvernance professionnelle de l'éco-

(11) Gestion prévisionnelle des emplois et des compétences, Secteur pêche, pays de Cornouaille, MARS 2009. nomie des pêches, à l'activation multiniveaux des interactions entre acteurs professionnels et acteurs publics. De ce fait, la trajectoire historique française de type étato-corporatiste disposerait au moins d'autant de facteurs propices au changement et à l'adaptation que de facteurs producteurs de rigidité ou d'immobilisme davantage couramment associés à cette forme institutionnelle.

Ces innovations institutionnelles contrastées et parfois contradictoires car inscrites dans des registres de légitimation différents ne naissent pas par hasard dans tel ou tel quartier. Elles sont au contraire congruentes avec les spécificités culturelles et institutionnelles des systèmes productifs localisés qui les portent et ne sont pas totalement exemptes d'enjeux de concurrences et de luttes symboliques entre ces mondes productifs (SALAIS, STORPER, 1993).

Ainsi dans les exemples retenus, le Quartier du Guilvinec nous paraît davantage porteur, en matière de conception du lien formation-emploi, du modèle d'intégration républicain, fondé sur l'enrichissement du secteur des pêches en emplois et en maind'oeuvre qualifiée. Ce modèle républicain peut aussi être étendu au champ de l'insertion professionnelle quand il s'agit d'initier des jeunes des banlieues au monde de la pêche afin de faciliter leur intégration sociale et professionnelle. Le CLPMEM du Guilvinec a imaginé l'organisation d'une classe «découverte des milieux de la pêche» à destination de jeunes issus des zones d'éducation prioritaire de banlieues de la région parisienne. Cette action devrait également être renouvelée en direction des jeunes des banlieues des villes cornouaillaises:

«On a accueilli une classe de $3^{e}$ de Mantes-la-Jolie
en classe de découverte. Une association de Mantes-
la-Jolie avait fait un travail assez extraordinaire pour
que les jeunes sortent du ghetto des communautés.
Donc, un car de 50 personnes est arrivé ici trois
semaines avant, pour voir où ils allaient laisser leurs
enfants! Le car arrive, multicolore, sensationnel!
Des boubous, des Africains, des Arabes, des femmes
voilées, des mecs au chapeau, des mecs habillés tout
en blanc [...]. Les jeunes ont fait du bateau école, un
peu de simulateur, un peu de ramendage, un peu de
voile l'après-midi, de la découverte du milieu marin!
Après, C. a rencontré Y. pour instituer ça de façon
régulière, routinière, pour des quartiers de proxi-
mité comme Quimper, Brest, Lorient, enfin des villes
bretonnes dans lesquelles il y a aussi des quartiers en
difficulté... et ainsi on a institué un module découverte
d'entreprise dans les classes de $3^{e}$ (12). »

Les quartiers de Bretagne nord qui doivent concilier les besoins en main-d'œuvre des segments de petite pêche et d'armements hauturiers coopératifs mettent l'accent sur la réactivation contemporaine de la formation par apprentissage à bord des navires

(12) Interview directeur du lycée maritime du Guilvinec. 
hauturiers et sur la création de groupements d'employeurs pour les côtiers proches du modèle des mondes interpersonnels (SAlais, STORPER, 1993) propres aux relations artisanales.

"Le système de l'apprentissage, c'est un temps en mer, un temps à terre. Pour la formation obligatoire à terre, il était quasiment impossible de coupler le rythme de travail et de vie des hauturiers qui rentrent tous les quinze jours avec celui des côtiers qui rentrent tous les jours. Du coup, l'expérience s'est bien développée pour les hauturiers des Côtes-d'Armor et par contre pour les côtiers, ça a moins bien fonctionné. Il fallait faire un choix entre un système et l'autre. Aussi, chez nous, l'idée se développe de faire des groupements d'employeurs pour les petits côtiers. Ils leur permettraient de se "partager» un apprenti tout en bénéficiant d'une souplesse administrative et favoriseraient l'usage des dispositifs de formation en alternance, d'apprentissage ainsi que les contrats de professionnalisation qui vont devenir très importants (13). »

Les armements semi-industriels concarnois confrontés aux difficultés de recrutement ont tenté de créer leur propre CAP par apprentissage en partenariat avec le Centre européen pour la formation professionnelle continue afin de former leur maind'œuvre sur un modèle plus proche du monde industriel (SALAIS, StoRPER, 1993) privilégiant davantage la standardisation des pratiques de travail.

Enfin, la diversité de ces initiatives exige des espaces de coordination, qu'ils soient de type horizontal ou vertical, pour s'articuler aux demandes d'emploi de la société globale. Il s'agit à la fois, pour la socio-économie des pêches maritimes de Bretagne, de se nourrir de la pluralité des initiatives produites par ses propres mondes productifs et de construire une gestion quantitative et qualitative des flux de demandeurs d'emploi en lien avec le Service public de l'emploi. La gestion qualitative des flux renvoyant plus particulièrement à la construction de dispositifs localisés d'insertion permettant la prise en compte des problématiques d'accompagnement en rapport avec les spécificités productives du milieu.

\section{L'appropriation des problématiques d'accompagnement vers l'emploi par le milieu: l'exemple de Cap Avenir}

L'appropriation des problématiques de l'accompagnement vers l'emploi par ce secteur en pénurie de main-d'œuvre repose sur une construction interactionniste des relations localisées d'insertion. En réactivant des modèles culturels traditionnels d'alternance du milieu dans le cadre des dispositifs

(13) Interview d'un permanent du Comité régional des pêches maritimes et des élevages marins de Bretagne. contemporains d'insertion, l'association de développement économique du secteur de la pêche en Bretagne nord, Cap Avenir, contribue à la production d'une instance de médiation (WuHL, 1996) entre système productif et système d'insertion. Cette figure professionnelle d'accompagnateur vers l'emploi dans le milieu des pêches associe étroitement les rôles d'accompagnateur des parcours d'insertion et de coordonnateur de partenariats contribuant, pour ce dernier, au renforcement du maillage socio-économique et institutionnel du milieu des pêches.

\section{L'accompagnement par le milieu dans le cadre des dispositifs publics d'insertion: une conception interactionniste de la relation entre systèmes productif et d'insertion}

Dans son ouvrage consacré aux politiques d'insertion, Simon WuHL (1996) établit une analyse critique approfondie des causes de la faible efficacité des politiques d'insertion en matière de lutte contre le chômage. Il examine la responsabilité partagée des dispositifs publics d'insertion et des entreprises dans la production d'un chômage à la française particulièrement résistant à la résorption du chômage de masse. Le point de rencontre entre ces deux causalités réside dans la nature de la relation établie entre le champ de la formation et de l'insertion, d'une part, et celui de l'entreprise, de l'autre. Cette relation est selon lui de type fonctionnaliste et adéquationniste alors que le défi du chômage d'exclusion exige que les relations entre les deux champs soient de type interactionniste. La conception fonctionnaliste de l'insertion trouve la source du chômage dans l'insuffisante employabilité du demandeur d'emploi. La réponse privilégiée est donc celle de la formation. Le problème de cette formule est la position d'extériorité de l'entreprise dans la relation de formation et d'insertion. De ce fait, rien ne garantit à l'issue de la formation que le demandeur d'emploi trouvera l'entreprise «adéquate» intéressée par son employabilité. Cette conception est issue selon Whul de la période fordiste de l'économie «reposant sur une division fonctionnelle autour de la relation emploi qui procède à une séparation entre le processus de mise à niveau de la force de travail (formation insertion élargie) d'une part et celui de son utilisation par la production d'autre part»; la réarticulation «adéquate» entre ces deux procès - de la formation de la main-d'œuvre, d'un côté, et de son usage par le système productif, de l'autre - s'opérant en partie grâce au système fordien de négociation professionnelle visant la production de grilles de classification mettant en correspondance les postes de travail et les qualifications. Il lui oppose une conception interactionniste de la relation d'insertion dans laquelle l'action de formation ou plus largement d'insertion fait l'objet d'une co-construction entre 
l'ensemble des acteurs du milieu productif et d'insertion local. L'entreprise se trouve alors en position endogène dans cette "relation d'insertion localisée». Nous considérons que selon cette conception, la construction des parcours d'insertion dans le cadre des dispositifs localisés d'insertion ne saurait être dissociée de la construction des partenariats qui peuvent être considérés comme les substrats relationnels de ces parcours d'insertion. De ce point de vue, les parcours d'insertion des demandeurs d'emploi et les réseaux partenariaux des dispositifs localisés d'insertion peuvent être considérés comme les deux faces d'une même réalité sociale notamment au stade expérimental de son élaboration. La première face renvoie aux interactions qui maillent le parcours d'insertion au stade expérimental de l'élaboration du dispositif d'insertion dont la reproduction et la répétitivité à terme contribueront, par la stabilisation du partenariat, à son institutionnalisation (BERGER, LUCKMANN, 1989).

\section{L'association Cap Avenir: la construction d'une instance de médiation entre système productif et système d'insertion dans la socio-économie des pêches maritimes en Bretagne nord}

L'association Cap Avenir a été créée en 2002 à l'initiative des comités locaux des pêches maritimes et des élevages marins, et de la coopération maritime de Bretagne nord. Elle s'est distinguée par ses actions dans le domaine du recrutement en tentant de résoudre les problèmes de pénurie de main-d'œuvre des flottilles de pêche de Bretagne nord et plus précisément des flottilles hauturières plus sensibles au problème(14). Pour ouvrir le milieu de la pêche maritime à des recrutements élargis, elle va réactiver le modèle, traditionnel dans le milieu, de l'alternance entre formation en établissement et formation sur le tas en s'inscrivant dans les dispositifs contemporains de l'apprentissage tant pour les diplômes de formation initiale que continue. La réactivation de ce modèle ancien suppose de construire simultanément les réseaux d'entreprises susceptibles d'accueillir les futurs marins pêcheurs en apprentissage et le partenariat institutionnel permettant d'alimenter le dispositif en candidats potentiels. Le cœur de cette activité simultanée d'accompagnement des candidats et de coordination du partenariat s'inscrit dans le rôle de l'accompagnateur-coordonnateur qui porte le rapport interactionniste entre le système d'insertion et le système productif. Cette relation interac-

(14) Le segment hauturier est particulièrement sensible à la pénurie de main-d'œuvre car la taille des équipages et les exigences de brevets sont nettement plus élevées qu'en pêche côtière et parce que les conditions de travail (alternance veille/ sommeil très fragmentée, conditions météo souvent difficiles) et de vie (marée de huit à quinze jours coupées par deux à trois jours à terre) sont réputées plus difficiles. tionniste repose notamment sur des négociations normatives entre représentants de l'univers sectoriel et représentants des institutions généralistes de la société globale. Elle pose également la question des conditions préalables au fonctionnement de ce dispositif d'insertion localisé : celle de sa coordination verticale (avec le service public de l'emploi) et horizontale (avec la socio-économie territoriale maritime).

\section{Réactiver le modèle traditionnel de l'alternance en s'inscrivant dans les dispositifs contemporains de l'apprentissage}

Les structures de gouvernance professionnelle et de formation maritime de Bretagne nord ont choisi de privilégier le recours aux dispositifs d'apprentissage sous deux formes: le CAP par apprentissage décerné en un an dans le cadre du lycée maritime de Paimpol et le contrat de professionnalisation dont le suivi et la mise en œuvre sont assurés par Cap Avenir. La mission de cette association est également élargie au suivi du dispositif du CAP par apprentissage en partenariat avec le lycée maritime. Ces deux mesures visent l'ouverture du recrutement au-delà des viviers du milieu. Le CAP par apprentissage (15) vise des publics scolaires déjà détenteurs d'un CAP d'une autre branche d'activité, ce qui évite une concurrence frontale avec les CAP classiques du milieu et favorise les mobilités d'autres secteurs d'activité vers la pêche. Le contrat de professionnalisation s'adresse aux demandeurs d'emploi de plus de 26 ans et aux jeunes de moins de 26 ans. Il prévoit l'organisation alternée de quinze semaines de formation en centre de formation et de cinq semaines d'embarquement. Le suivi de ces deux dispositifs met Cap Avenir au défi de construire un réseau d'intermédiaires de l'emploi pour orienter les candidats vers l'association et de patrons pêcheurs pour les accueillir.

«Mes partenaires ce sont: le lycée maritime, les
comités locaux des pêches, toutes les instances
emploi: mission locale, ANPE. Le partenariat, c'est
d'être partout tout le temps et au fait de tout ce qui
se passe. Avec le directeur, je l'appelle, on n'arrête
pas de se téléphoner. Je suis avec les référents des
comités locaux le plus possible, enfin fréquemment, à
des réunions. Je ne vois pas d'autres façons d'institu-
tionnaliser un partenariat que d'être là au moment où
il faut y être(16).»

Sa structure associative au service de la socioéconomie territoriale de la Bretagne nord la dispose à représenter le système productif localisé tandis que sa fonction de suivi des dispositifs d'alternance en fait l'interlocuteur privilégié des composantes élargies du SPE.

(15) L'obtention du diplôme se fait en un an au lieu de deux pour des CAP classiques à la condition que les candidats soient déjà détenteurs d'un $\mathrm{CAP}$ dans un autre secteur.

(16) Interview du responsable de l'association Cap Avenir. 


\section{Le rôle de l'accompagnateur-coordinateur: construction simultanée des parcours et du partenariat au stade expérimental}

La mise en oeuvre du contrat de professionnalisation focalise sur l'accompagnateur-coordonnateur l'ensemble des interactions qui constitue le système d'accompagnement: celles qui le relient au public, aux prescripteurs qui alimentent le dispositif et aux utilisateurs qui accueillent et forment les bénéficiaires. Plus globalement, l'élargissement du champ de l'accompagnement vers l'emploi aux aspects d'insertion sociale (santé, habitat, transport, autonomie financière, difficultés diverses), de formation, et d'accès à l'entreprise, exige de l'opérateur la construction d'un système d'acteurs, extrêmement large, reliés entre eux par la construction du parcours d'insertion.

«L'accompagnement vers l'emploi, c'est du premier contact avec la personne jusqu'au moment où elle signe son contrat; l'accompagnement dans l'emploi, c'est toute la période où elle est dans son contrat de travail jusqu'au moment où elle est insérée professionnellement. C'est ce que je fais tous les jours... C'est le type qui m'appelle, il peut pas se déplacer, il a plus d'essence dans sa voiture, c'est aussi simple que ça. Je trouve une carte... il a pas d'argent... j'appelle la mission locale qui fait passer à la commission spéciale, lui obtient $200 €$, etc. Vous voyez, c'est trouver une solution à son problème aujourd'hui(17). »

Au stade expérimental de la mise en œuvre des dispositifs, l'accompagnateur doit veiller à tous les écueils qui interrompent la continuité du parcours. Il doit restaurer par les interactions pertinentes les substrats relationnels qui assurent le portage des parcours d'insertion, c'est-à-dire l'accompagnement par le milieu. C'est dans ce sens que l'on peut parler d'effet de construction collective de l'accompagnement individuel ou de contribution des dispositifs localisés d'insertion au maillage socio-institutionnel de l'économie des pêches.

«Par exemple, on met les contrats de professionnalisation en place, mais si je ne vais pas faire remplir les contrats, arranger le rendez-vous entre le jeune et l'entreprise, si je ne suis pas le cheminement du contrat, ça s'égaye dans la nature. Il faut quelqu'un qui porte ça de $A$ à $Z$ et qui convainque les gens aussi (18).»

«On va parler d'une politique concertée d'emploi-formation mais si dans tel port, il n'y a pas les moyens, donc je pense qu'il faudrait aussi réfléchir à ça, un maillage qui tienne la route, des partenariats mais qui tiennent la route et puis qui s'adaptent à la réalité portuaire (19).»

\section{Les modes interactionnistes de coordination entre institutions sectorielles et institutions globales}

Dans l'expérience de Cap Avenir, le travail de coordination entre acteurs institutionnels, repose, au stade expérimental de la mise en oeuvre des dispositifs d'accompagnement, sur la position particulière de l'accompagnateur-coordonnateur. Il constitue une sorte de «marginal sécant» (CROzIER, 1963), pris entre deux mondes, à la fois sur un plan biographique et sur un plan relationnel, et assurant le travail de médiation entre le monde des pêches maritimes et celui des institutions générales de l'emploi.

«Je suis théoricien de départ, j'ai fait un doctorat de philosophie politique. Je suis aussi un praticien puisque j'ai été recruté parce qu'on cherchait quelqu'un d'expérimenté en pêche et cultures marines. Ce qui est très paradoxal. Je connais le secteur, j'ai été responsable pêche, j'ai travaillé pour les cultures marines, j'ai été manouvre ostréicole, j'ai été marin professionnel embarqué, même si c'était pas à la pêche. Oui, je pense que j'avais parfaitement le profil pour avoir la confiance des patrons de pêche. Ils ont besoin d'avoir confiance dans les gens (20)! »

$\mathrm{Au}$ stade expérimental, il est l'opérateur du rapport interactionniste entre les deux mondes qui ne se rencontrent pas nécessairement. Il doit pouvoir être le traducteur des attentes et modes de fonctionnement des marins pêcheurs auprès des intermédiaires publics de l'emploi tout en veillant à la bonne compréhension par les marins pêcheurs des stocks de connaissance de l'univers administratif. Il cherche à établir une prise en considération mutuelle, des valeurs et normes culturelles, propres à chaque monde.

«Les marins sont des gens qui peuvent donner tout
ou se fermer comme une huître et ça joue sur très
peu de chose. Il faut jouer franc jeu, je veux dire il
faut être vraiment franc du collier, parler nettement
et traiter tout nettement, et ils ont pas de pratiques
de l'ANPE... vous voyez? Ce sont deux mondes qui
s'ignorent. Quand je dis aux pêcheurs que je vais
déposer une offre... ils rigolent... D'abord parce que
l'ANPE n'envoient pas les bonnes personnes, ensuite
parce qu'ils ne la connaissent pas, ensuite parce qu'il
y a des préjugés... réciproques peut-être (21).»

Toutefois ce marginal sécant appartient à la socio-économie des pêches. De ce fait, le travail de coordination porte la coloration particulière de ce milieu. Ainsi le travail d'acculturation des intermédiaires publics de l'emploi aux pratiques partenariales du milieu des pêches va s'opérer, par le recours systématique, initié par l'accompagnateurcoordonnateur, à des modèles de relations typiques du milieu. Il affirme fort sa préférence pour les relations fondées sur l'oralité, le face à face, l'in- 
terconnaissance personnelle et la confiance, propres à cette économie de la proximité. En mobilisant les ressources culturelles du milieu des pêches, il crée les conditions de l'innovation institutionnelle dans le champ des dispositifs publics d'accompagnement. En favorisant les relations de face à face et d'interconnaissance entre les acteurs publics de l'emploi et de la socio-économie des pêches, il favorise l'invention de nouveaux modes de coordination entre institutions fondés sur les nouveaux modèles de relations construits entre ces acteurs.

\begin{abstract}
"Quand je suis arrivé, j'ai commencé par rendre visite à toutes les missions locales, à tous les CIO. J'ai distribué des plaquettes, communiqué, et je me souviens avoir rencontré une directrice de mission locale, directrice adjointe, qui m'a dit: "Mais, Monsieur, pourquoi êtes-vous venu?", genre: "C'était pas utile, fallait faire porter par..." Je lui ai dis: "Mais, Madame, parce que je vous vois, parce que je vous connais, parce que physiquement je sais qui vous êtes et parce que je pense qu'un problème d'emploi se résoudra par des contacts humains, et la connaissance par un réseau, et un réseau, c'est pas un réseau au téléphone, c'est un réseau de gens qui se connaissent." Et, donc j'essaye de faire un maillage au niveau de toutes ces instances, au niveau de l'emploi et un maillage un peu officieux au niveau de la formation(22).»
\end{abstract}

\section{Coordination territoriale et sectorielle du dispositif localisé d'insertion}

Cette intégration interactionniste fondée sur l'influence mutuelle entre les acteurs se pose à deux niveaux: horizontaux, c'est-à-dire territoriaux, et verticaux, c'est-à-dire entre les institutions sectorielles et globales du champ de l'emploi. L'intégration horizontale met les acteurs des socio-économies des pêches au défi d'articuler la grande diversité de leurs innovations locales, imaginées pour ouvrir leurs marchés du travail à la société globale, en un modèle d'ensemble. Il les met au défi de dépasser les dimensions parfois concurrentielles liées à la confrontation des spécificités institutionnelles et culturelles locales pour en faire des ressources mutualisables pour l'ensemble des quartiers maritimes.

"Effectivement avant la crise de 1994, il y avait des singularités portuaires mais aujourd'hui on arrive à une situation où le secteur a encore diminué... l'heure des querelles portuaires est passée. Il faut qu'on tire tous dans le même sens. Le comité régional a tout à fait ce discours-là! Il faut qu'on communique de façon plus concertée. Il faut qu'on trouve du monde. Il faut qu'on intéresse un public qui n'est pas forcément maritime. C'est pour ça qu'il faudrait faire une espèce de bilan. Moi, je me suis inspiré de ce qui se faisait dans le sud par exemple avec le CIP, en me disant pourquoi le CIP n'existe pas dans le nord. Donc cherchons un rapprochement qui peut passer par le comité régional(23).»

(22) Idem.

(23) Idem.
La signature d'une convention régionale entre l'agence régionale de l'emploi, le comité régional des pêches maritimes et des élevages marins et le syndicat régional de la conchyliculture va indéniablement dans ce sens. Elle favorise l'établissement territorialisé de partenariats stratégiques et opérationnels entre les acteurs pertinents du secteur des pêches et du SPE. Elle crée les conditions de l'établissement d'instances de médiation localisées, propres au secteur, entre système productif et système d'insertion.

«L'enjeu là c'était d'arriver à mettre en phase les professionnels et l'ANPE, donc, on a décidé de mettre en place des personnes ressources. C'est pas forcément chaque comité local, mais au moins dans chaque département, pour les structures qui le souhaitent, signer des conventions locales entre ALE et les comités locaux(24). »

«Depuis que la convention cadre est signée, je ne sais pas s'il y a une relation de cause à effet mais je pense que oui, j'ai beaucoup plus d'appels: la maison de l'emploi de Nantes, par exemple me téléphone pour me dire: "J'ai eu votre contact par les Affaires maritimes..." Les Affaires maritimes me téléphonent: "J'ai un patron et un marin dans le bureau qui sont intéressés, est-ce que vous pouvez faire signer un contrat de professionnalisation." Vous voyez, ce n'est plus du bouche à oreille (25).»

L'intégration verticale pose le problème des modes d'articulation entre gestion quantitative et qualitative des flux ainsi que des modes de division du travail d'accompagnement entre le SPE et les intermédiaires associatifs ou privés. La mise en correspondance des marchés du travail des pêches maritimes, d'une part, et des flux quantitatifs des demandeurs d'emploi gérés par l'ANPE et les Assedic, d'autre part, constitue un enjeu majeur.

\begin{abstract}
"Avant la convention cadre de l'ANPE, mon problème c'est qu'il fallait que j'arrive à convaincre le directeur de l'ANPE de St.-Brieuc à chaque fois et le message, il fallait que je le répète chaque fois. Là, les agents en Bretagne sont au courant en temps réel. Ca peut être une directive et ça passe, et c'est déterminant. Je me suis dit, si on arrive à utiliser l'outil de l'ANPE qui est un gros outil, qui n'est pas forcément totalement adapté, mais c'est très puissant, je suis sûr qu'on va avoir des résultats (26).»
\end{abstract}

Pour qu'elle s'établisse, elle suppose une identification claire des métiers de la pêche dans les classifications propres à ces institutions qui restent encore assez largement à construire.

«On ne veut pas, nous, faire de tous les élèves, des
conchyliculteurs ou des pêcheurs, on veut simplement
que sur 1000 élèves, il y en ait 8 qui sachent que ça
existe... ils sont en train de faire le travail [de codifi-
cation]... c'est très complexe, mais en tout cas, quand

(26) Idem. 
on n'a pas bien identifié les métiers, on ne recrute pas correctement (27).»

Elle suppose aussi que l'information sur ces métiers soit disponible dans les centres d'information et d'orientation et relayée par les professionnels de l'enseignement, de l'orientation et du placement. $\mathrm{Si}$ ces initiatives peuvent garantir l'orientation de flux exogènes au milieu vers le secteur des pêches elle ne permet pas la discrimination qualitative des flux auparavant favorisée par la socialisation interne au milieu.

"Les missions locales font leur métier mais, plus en amont, au niveau des centres de formation et d'orientation dans les lycées et collèges, la pêche et les cultures marines sont les deux seuls métiers qui ne sont pas identifiés. Mais il ne suffit pas de codifier. Le problème après, c'est qu'il faudrait une période transitoire d'essai des jeunes, qu'ils soient embarqués pour une journée en marée pêche ou même une journée sur le bateau école qui a été acheté par le lycée de Paimpol, pour voir leur réaction. Le type qui est malade tout de suite, ce n'est pas la peine de monter un contrat si vraiment physiquement, il ne le supporte pas(28).»

Les dispositifs d'insertion professionnelle et d'accompagnement initiés ou adaptés par le milieu peuvent favoriser cette gestion qualitative.

"Effectivement, il y a d'un côté la grosse machine de l'ANPE et de l'autre côté, les bonnes pratiques porto-portuaires des professionnels. Les jeunes me disent: "Mais moi j'ai peur d'aller sur les bateaux!" Ils prennent les marins pour une secte! Et moi je les introduis. C'est pas du tout évident pour un jeune qui se retrouve au Guilvinec avec un patron de pêche qui a 30 ans de carrière! C'est pire que de partir dans un ashram tibétain quoi! Enfin, j'exagère! Je veux dire c'est une expérience, il faut quelqu'un qui soit là... pour faire le lien(29). »
L'appropriation de la problématique de l'accompagnement vers d'emploi par le secteur des pêches maritimes nous a permis d'interroger la face cachée de l'accompagnement: celle de la construction institutionnelle des maillages favorisant l'accès des demandeurs d'emploi extérieurs au secteur à ces socio-économies particulières. Si la problématique de l'accompagnement interroge habituellement les conditions singulières d'orientation des chômeurs dans le monde de l'emploi, elle interroge tout autant les modes de construction de ces substrats relationnels entre système d'insertion et systèmes productifs. Dans cette perspective, le renforcement des capacités d'orientation autonome du chômeur dans le champ de l'emploi, attendu des dispositifs d'accompagnement, dépend au moins autant de la construction des maillages socio-institutionnels entre univers productifs et champ de l'insertion que des caractéristiques singulières du parcours du demandeur d'emploi. Une telle démarche suppose de considérer l'emploi non comme une réalité donnée que le demandeur d'emploi doit trouver et à laquelle il doit s'adapter mais comme une construction interactionniste située mettant en relation les acteurs multiples que sont les demandeurs d'emploi, les systèmes productifs et les systèmes d'insertion considérés. 


\section{Bibliographie}

Bagnasco A., Trigilia C. (1988), La construction sociale du marché: le défi de la troisième Italie, Cachan, Ed. de l'ENS Cachan, coll. «Sciences sociales».

Bazalni B., Béraud M., Boulayoune A., Divay S., Eydoux A., GouzIEn A. (2008), Accompagnement vers l'emploi, Acteurs, pratiques, dynamiques, Rapport final pour la Dares, avril.

Berger P., LuCKMANn Th. (1989), La construction sociale de la réalité, Paris, Méridiens Klincksieck.

CÉREQ/ Bretagne, PoDEvin G. (coord.) (2003), Le secteur des pêches maritimes, pour le compte du ministère des Affaires sociales, du Travail et de la Solidarité, et de la Délégation générale à l'emploi et à la formation professionnelle, Paris, La Documentation française.

Courlet C., Pecquert B. (1992), «Les systèmes industriels localisés en France: un nouveau modèle de développement», in Benko et Lipietz A. (dir.), Les régions qui gagnent, Presses universitaires de France, pp. 81-102.

Crozier M. (1963), Le phénomène bureaucratique, Paris, Seuil.

Gouzien A. (1997), Les marins pêcheurs du Guilvinec, systèmes d'emploi, mobilités et identités socioterritoriales, rapport de recherche pour le compte de la Dares, «Autres façons de travailler, autres mœurs, identités sociales des groupes en situation particulières d'emploi ».

Gouzien A. (2003), «Monographie: la zone de Concarneau », in CÉREQ/ Bretagne, Gérard Podevin (coord.), Le secteur des pêches maritimes, pour le compte du ministère des Affaires sociales, du Travail et de la Solidarité, et de la Délégation générale à l'emploi et à la formation professionnelle, Paris, La Documentation française.

Gouzien A. (2004), «Pénurie de marins et carrières professionnelles à la pêche maritime: identités professionnelles et régulations institutionnelles locales de l'activité de pêche maritime», in L'observatoire des droits des marins, Nantes, 22-23 janvier 2004.

Gouzien A. (2005a), «Les coopératives maritimes dans les dynamiques de territorialisation de la socio-économie des pêches cornouaillaises », in Xabier Itcaina, Jacques Palard, Sébastien Ségas, Régimes territoriaux et dévelop- pement économique, Rennes, PUR, 2007.

Gouzien A. (2005b), «Pêche maritime en Bretagne sud: identités socio-économiques de quartier», Cahier de $l^{\prime} A R S, \mathrm{n}^{\circ} 2, \mathrm{UBO}$.

GouzIen A. (2008), «Pratiques et institutions anciennes et contemporaines de l'accompagnement vers l'emploi dans le secteur des pêches maritimes en Bretagne », in Accompagnement vers l'emploi, Acteurs, pratiques, dynamiques, Bernard Bazalni, Mathieu Béraud, Ali Boulayoune, Sophie Divay, Anne Eydoux, Gouzien A., Rapport final pour la Dares, avril 2008.

Le Treust N. (2007), Le secteur des pêches maritimes et l'accompagnement dans l'emploi: le cas des quartiers maritimes de Paimpol et St Brieuc, Mémoire de Master-2 formation, insertion, Dir. A. Gouzien, octobre.

Levesque B., Bourque G.-L., Forgues E. (2001), La nouvelle sociologie économique, Paris, Desclée de Brouwer (Sociologie économique).

Mordrel L. (1972), Les institutions de la pêche maritime: histoire et évolution: essai d'interprétation sociologique, Thèse pour le doctorat de droit, Université de droit, d'économie et de sciences sociales de Paris (Paris 2), décembre.

Paradeise C., Vourch F. (1982), «La marine marchande: du système corporatiste au système occupationnel». Extension et segmentation du marché du travail maritime, in Travailleurs des transports et changements technologiques: résultats des recherches en sciences humaines, colloque de Versailles, Palais des congrès, juin 1982.

Salais R., Storper M. (1993), Les mondes de production: enquête sur l'identité économique de la France, Paris.

TRIPIER P. (1991), Du travail à l'emploi: paradigmes, idéologies et interactions, Ed. de l'Université de Bruxelles.

WuHL S. (1996), Insertion: les politiques en crise, Paris, PUF. 